\title{
Self-Service-Portale für das IT-Service-Request-Management: Identifikation relevanter Use-Cases
}

\author{
Sebastian Floerecke (iD
}

Eingegangen: 13. August 2021 / Angenommen: 2. Oktober 2021 / Online publiziert: 10. November 2021 (C) Der/die Autor(en) 2021

Zusammenfassung Unternehmen sind im Rahmen ihrer Digitalisierungsvorhaben vermehrt auf der Suche nach einem ihrer IT-Service-Management(ITSM)-SoftwareSuite ergänzenden Self-Service-Portal. Damit sollen sich Mitarbeiter über die im Service-Katalog enthaltenen IT-Services informieren und eigenständig unterschiedliche Service-Request-Arten auslösen können. Die Self-Service-Portal-Auswahl und -Einführung stellen Unternehmen jedoch vielfach vor eine Herausforderung. Als Gründe gelten insbesondere die hohe Komplexität von ITSM-Software-Suiten, deren vielschichtige Verzahnung innerhalb der Unternehmensarchitektur und der große, intransparente Tool-Markt. Zudem weisen die erhältlichen Self-Service-Portale im Standard gegenwärtig mehrheitlich einen relativ niedrigen Reifegrad auf. Die Forschung befindet sich im Bereich der Self-Service-Portale im Allgemeinen und bezüglich deren notwendigem Funktionsumfang im Besonderen noch relativ am Anfang und trägt somit wenig zur Problemlösung bei. Vor diesem Hintergrund geht vorliegender Beitrag im Rahmen einer explorativen Einzelfallstudie innerhalb eines Großunternehmens der Frage nach, welche Use-Cases (Service-Request-Typen) ein Self-Service-Portal abbilden sollte. Zentrales Ergebnis ist ein aus 16 Use-Cases bestehender Katalog, der als Referenz gleichermaßen für anbietende und einführende Unternehmen dienen kann. Der Beitrag zeigt, dass Mitarbeiter vielfältige Anliegen haben, die weit über die Neubestellung und Kündigung von IT-Services hinausgehen. Diesem Umstand sollte ein Self-Service-Portal gerecht werden.

Schlüsselwörter Self-Service-Portal · Service-Request-Management · Use-Cases · IT-Service-Katalog $\cdot$ Explorative Einzelfallstudie

\footnotetext{
Sebastian Floerecke $(\bowtie)$

Lehrstuhl für Wirtschaftsinformatik mit Schwerpunkt Informations- und IT-Service-Management, Universität Passau, Innstraße 43, 94032 Passau, Deutschland

E-Mail: sebastian.floerecke@uni-passau.de
} 


\title{
Self-Service Portals for IT Service Request Management: Identification of Relevant Use Cases
}

\begin{abstract}
As part of their digitization initiatives, companies are currently increasingly looking for a supplementary self-service portal for their IT service management (ITSM) software suites. Self-service portals allow employees to obtain information about the IT services contained in the service catalog and to independently trigger different service request types. However, selecting and introducing a self-service portal often poses a challenge for companies. The reasons are in particular the high complexity of ITSM software suites, their multi-layered interlocking within the enterprise architecture and the large, non-transparent tool market. In addition, the majority of the self-service portals available show a relatively low level of maturity. Research in the field of self-service portals in general and regarding their necessary range of functions in particular is still relatively early and therefore contributes little to solve the problem. Beyond this background, this article examines the question of which use cases (service request types) a self-service portal should cover by conducting an exploratory single-case study within a large company. The central result is a catalog consisting of 16 use cases, which can serve as a reference for both supplying and introducing companies. The article shows that employees have a wide range of concerns that go well beyond ordering and terminating IT services. A self-service portal should take this into account.
\end{abstract}

Keywords Self-Service Portal · Service Request Management · Use Cases · IT Service Catalogue · Exploratory Single-Case Study

\section{Problem- und Zielstellung}

Zur Erbringung der verschiedenen IT-Service-Management(ITSM)-Prozesse, wie Demand-, Incident- und Service-Request-Management, setzen Großunternehmen und reifere KMUs branchenübergreifend komplexe ITSM-Software-Suiten (Beispiele: Cherwell, EasyVista und ServiceNow) ein (Floerecke 2020). Im Rahmen ihrer Automatisierungs- und Digitalisierungsvorhaben suchen sie gegenwärtig vermehrt nach einem darauf aufbauenden, ergänzenden Self-Service-Portal (Floerecke 2021a). Dabei handelt es sich um eine Softwareanwendung, mit deren Hilfe sich Mitarbeiter der eigenen oder einer anderen Organisation primär über die im IT-Service-Katalog enthaltenen atomaren (Beispiele: virtueller Server und Standard-Office-Software) und gebündelten IT-Services (Beispiele: Desktop- und mobiler Arbeitsplatz) informieren und Service-Requests wie Neubestellung und Kündigung auslösen können. Oftmals lassen sich damit weitere ITSM-Objekte wie Incidents und Demands erstellen und verfolgen (Abdelkebir et al. 2017; Doheny et al. 2020). Im Mittelpunkt steht, Personen mit dem für ihre Arbeitsaufgaben notwendigen IT-Equipment (Hard- und Software) auszustatten und bestmöglich mittels IT-Services zu unterstützen (Floerecke 2021b). Unternehmen und deren IT-Abteilungen versprechen sich vom Einsatz eines Self-Service-Portals insbesondere eine Entlastung der IT-Mitarbeiter von monotonen Routinetätigkeiten, Einsparungen von personellen Ressourcen und eine 
Reduzierung von Prozessdurchlaufzeiten (Lohse 2016). Die Akzeptanz derartiger Systeme gilt als hoch, da Mitarbeiter es ohnehin aus ihrem privaten Umfeld gewohnt sind, Produkte und zunehmend auch Dienstleistungen eigenständig in WebShop-Systemen rund um die Uhr zu bestellen (Schulz 2015). Durch die Einführung einer Self-Service-Technologie verändert sich die Rolle von Kunden grundlegend vom passiven Empfänger hin zum aktiven Teilnehmer im Service-Bereitstellungsprozess (Scherer et al. 2015).

Die Auswahl, die prozessuale und systemische Integration sowie das Customizing von Self-Service-Portalen stellen Unternehmen aber vielfach vor eine Herausforderung (Floerecke 2020, 2021b; Pröhl und Zarnekow 2019). Als Gründe gelten einerseits die hohe Komplexität von ITSM-Software-Suiten und abzubildenden ITSMProzessen, deren vielschichtige Verzahnung innerhalb der Unternehmensarchitektur mit einer Vielzahl von Schnittstellen zu Altsystemen und zuliefernden Systemen und andererseits der große, aus über 400 Anbietern bestehende und damit intransparente Tool-Markt (Floerecke 2021a). Hinzu kommt, dass es vielen Unternehmen Schwierigkeiten bereitet, einen vollständigen, adäquat strukturierten und beschriebenen IT-Service-Katalog, die Basis eines Self-Service-Portals, zu erstellen (Nord et al. 2016; Schorr und Hvam 2018). Bei einer vom Tool-Hersteller USU und dem Trainingsanbieter SERVIEW im Jahr 2019 in der DACH-Region gemeinsam durchgeführten Online-Umfrage (Bause und Landis 2019) gaben lediglich $14 \%$ der 140 teilnehmenden Unternehmen unterschiedlicher Größe an, über einen IT-ServiceKatalog zu verfügen, der eine Auflistung und Beschreibung aller angebotenen ITServices beinhaltet. $30 \%$ besitzen demnach keinen und $56 \%$ einen nur teilweise dokumentierten IT-Service-Katalog.

Die Forschung befindet sich auf dem Gebiet der Self-Service-Portale im Allgemeinen (Lohse 2016, Scherer et al. 2015) und bezüglich deren notwendigem Funktionsumfang im Besonderen in einem frühen Stadium (Abdelkebir et al. 2017; Floerecke 2020, 2021a; Nord et al. 2016). Die Information Technology Infrastructure Library (ITIL) - die führende Sammlung von Leitlinien, Schulungen und Zertifizierungen für die ITSM-Domäne - liefert in dieser Hinsicht ebenfalls keine Empfehlungen (Axelos 2019; Beims und Ziegenbein 2014). Die Hersteller verweilen größtenteils noch inmitten einer Experimentierphase, die durch stetiges Lernen und Weiterentwickeln gekennzeichnet ist (Betz und McKeon-White 2019). So weisen zahlreiche Self-Service-Portale im Standard einen relativ niedrigen Reifegrad auf und lassen damit Potenzial ungenutzt.

Angesichts der nicht zufriedenstellenden Gesamtsituation in Forschung und Praxis geht dieser Beitrag der Frage nach, welche Use-Cases (Service-Request-Typen) ein Self-Service-Portal im Bereich IT-Service-Request-Management abbilden sollte. Bislang liegen hierzu zwar einzelne wissenschaftliche Publikationen vor (Floerecke 2021a, b; Schopf 2017; Schulz 2015), diese schlagen allerdings lediglich wenige Basis-Use-Cases vor, ohne sie näher zu beschreiben und zu erläutern. Zur Beantwortung der Forschungsfrage wurde eine explorative Einzelfallstudie innerhalb eines deutschen Großunternehmens durchgeführt, das im Rahmen eines Projekts ein geeignetes Self-Service-Portal mit Schwerpunkt IT-Service-Request-Management als Erweiterung ihrer bestehenden ITSM-Software-Suite auswählt und einführt. Dabei wurden zahlreiche Workshops und Einzelinterviews mit internen ITSM-, Vertriebs- 
und IT-Service-Katalog-Spezialisten, späteren Anwendern sowie Herstellern und Dienstleistern von ITSM-Software-Suiten und Self-Service-Portalen abgehalten und iterativ Anforderungen, insbesondere den Use-Cases betreffend, erhoben und spezifiziert.

Der Beitrag ist folgendermaßen aufgebaut: Nachdem in Kap. 1 die Problemund Zielstellung dieser Untersuchung erläutert wurde, befasst sich Kap. 2 mit den Grundlagen von ITSM, IT-Service-Katalogen und Self-Service-Portalen und zeigt den aktuellen Forschungsstand auf. Kap. 3 beschreibt das forschungsmethodische Vorgehen. In den Kap. 4 und 5 werden die Untersuchungsergebnisse vorgestellt und diskutiert. Am Ende geht der Beitrag auf den Nutzen der Ergebnisse für Praktiker und Forscher, Limitationen und weiteren Forschungsbedarf ein.

\section{Grundlagen und Stand der Forschung}

Zur Umsetzung von ITSM in Unternehmen bietet die wissenschaftliche Literatur unterschiedliche Rahmenwerke und Standards. Hierzu zählen unter anderem ISO/IEC 20000 und das IT-Service-Capability-Maturity-Model. Am weitesten in der Praxis verbreitet und seit Längerem als De-facto-Standard gilt ITIL (Macias und Alonso 2019). ITIL definiert ITSM als ,[e]ine Reihe spezialisierter Fähigkeiten einer Organisation zur Generierung eines Werts für Kunden in Form von Services“ (Axelos 2019). Das übergeordnete Ziel von ITSM besteht demnach darin, die vorhandenen Fähigkeiten und Ressourcen derart zu managen, dass die Gestaltung der IT-Services optimal auf die Anforderungen der Kunden ausgerichtet ist (Beims und Ziegenbein 2014).

ITIL umfasst 34 Managementpraktiken, eine davon ist das Service-Katalog-Management. Dessen Hauptziel ist die Bereitstellung und Pflege eines vollständigen IT-Service-Katalogs (Axelos 2019). Der IT-Service-Katalog eines Unternehmens ist eine Aufstellung und Beschreibung, die transparent machen soll, welche IT-Services zu welchen Merkmalsparametern und zu welchem Preis von Mitarbeitern der eigenen (interne Kunden) und/oder einer anderen Organisation (externe Kunden) bezogen werden können (Macias und Alonso 2018). IT-Service-Kataloge schaffen eine gemeinsame Kommunikationsgrundlage zwischen Anbieter und Nachfrager und fungieren als Basis weiterer Managementpraktiken beziehungsweise ITSM-Prozessen wie dem Service-Request-, Incident- und Configuration-Management sowie von Teilen des betrieblichen Rechnungswesens (Schorr und Hvam 2018).

Bei IT-Service-Katalogen werden häufig zwei Sichten, eine Kunden- und eine interne IT-Sicht, unterschieden. Diese dienen jeweils unterschiedlichen Zwecken und erfordern daher spezifische Informationen (Nord et al. 2016; Tiemeyer 2020): Bei der Kundensicht werden IT-Services, die sogenannten Business-Services, in der Sprache der Kunden mittels einer Vielzahl von Attributen wie beispielsweise Name, Beschreibung, Leistungsbestandteile, Service-Level-Agreements und Preisinformationen beschrieben. Um seitens der IT den Kunden die Business-Services erbringen zu können, werden Technische Services (interne IT-Sicht) benötigt. Technische Services werden einerseits mit Business-Services und andererseits mit den für ihre Erbringung nötigen Configuration-Items (CIs), wie Anwendung-, Server- 
und Datenbankinstanzen, verknüpft. Die interne IT-Sicht umfasst zudem die Definition von Genehmigungsprozessen und zur Beschaffung und Entsorgung von ITKomponenten notwendige Aktivitäten (Nord et al. 2016; Tiemeyer 2020).

Verschiedene Untersuchungen zeigen, dass bei Weitem nicht allen Unternehmensund IT-Verantwortlichen die Bedeutung beider Sichten und deren notwendigen engen Verzahnung bewusst ist und deshalb oftmals nur eine davon umgesetzt wird (Macias und Alonso 2018). An dieser Stelle wird angemerkt, dass für die Beschreibung - die Festlegung und Befüllung der notwendigen Attribute - beider Sichten des IT-Service-Katalogs in der Literatur divergierende Ansätze existieren, die einer Zusammenführung und gegebenenfalls Erweiterung bedürfen. Nicht nur die Strukturierung eines IT-Service-Katalogs, sondern auch die Beschreibung einzelner ITServices bereitet vielen Praktikern Schwierigkeiten (Floerecke 2020; Pröhl und Zarnekow 2019).

Auf Basis der Kundensicht des IT-Service-Katalogs können Besteller ServiceRequests initiieren. Dies kann traditionell per Telefon oder E-Mail, oder eben über ein Self-Service-Portal, erfolgen (Floerecke 2020; Jäntti 2013). Bei Self-ServicePortalen der ITSM-Domäne handelt es sich um eine Softwareanwendung, mit deren Hilfe sich Mitarbeiter der eigenen oder einer anderen Organisation primär über die im IT-Service-Katalog enthaltenen atomaren (Beispiele: virtueller Server und Standard-Office-Software) und gebündelten IT-Services (Beispiele: Desktop- und mobiler Arbeitsplatz) informieren und Service-Requests auslösen können. Oftmals lassen sich damit weitere ITSM-Objekte wie Incidents und Demands erstellen und verfolgen (Abdelkebir et al. 2017; Brooks und Curti 2014). Über die Jahre hat sich ein breites Marktangebot an einerseits in ITSM-Software-Suiten integrierten Modulen und andererseits Stand-Alone-Lösungen entwickelt (Doheny et al. 2020). Eng verwandte Tool-Kategorien, die oberflächlich betrachtet ähnliche Funktionalitäten umfassen, wie insbesondere generische Shop-Systeme, stellen aufgrund deren großen Distanz zur ITSM-Domäne und deren aufwendigen Integration mit einer ITSMSoftware-Suite keine Alternative dar (Floerecke 2021a). Nach dem US-amerikanischen Marktforschungs- und Beratungsunternehmen Gartner (Doheny et al. 2020) wächst der globale ITSM-Tool-Markt kontinuierlich - zwischen 2010 und 2019 von 1,3 auf 4,3 Mrd. US-Dollar. Die drei Anbieter BMC, Ivanti und ServiceNow decken zusammen $70 \%$ des Marktvolumens ab. Auch wenn es in den vergangenen Jahren zu einzelnen Übernahmen und Produktablösungen gekommen ist, spricht Gartner nicht von signifikanten Konsolidierungstendenzen.

Die Forschung befindet sich auf dem Gebiet der Self-Service-Portale im Allgemeinen (Lohse 2016; Scherer et al. 2015) und bezüglich deren notwendigem Funktionsumfang im Besonderen in einem frühen Stadium (Abdelkebir et al. 2017; Floerecke 2020, 2021a; Nord et al. 2016). ITIL bietet in dieser Hinsicht keine Gestaltungsempfehlungen (Axelos 2019; Beims und Ziegenbein 2014). Auch die Hersteller sind größtenteils noch mitten in einer Experimentierphase, die durch stetiges Lernen und Weiterentwickeln gekennzeichnet ist (Betz und Mckeon-White 2019). Demnach weisen gegenwärtig viele Self-Service-Portale im Standard einen relativ niedrigen Reifegrad auf und lassen damit Potenzial ungenutzt. Dieser Umstand macht projektspezifische Customizing-Maßnahmen oftmals unerlässlich. Die daraus gewonnenen Erkenntnisse fließen wiederum in die schrittweise Weiterentwicklung 
der Tools. Die wenigen vorhandenen wissenschaftlichen Publikationen (Floerecke 2021a, b; Schopf 2017; Schulz 2015) schlagen jeweils einzelne Basis-Use-Cases vor, ohne diese näher zu beschreiben und zu erläutern. Dieser Beitrag befasst sich deshalb mit der Frage, ob diese für Mitarbeiter in ihrem Arbeitsalltag ausreichend sind. Denn ein Self-Service-Portal sollte sich grundsätzlich an den Bedürfnissen und der Denk- und Arbeitsweise der Anwender orientieren (Mann 2017).

\section{Forschungsdesign}

Zur Ermittlung der seitens eines ITSM-Self-Service-Portals abzudeckenden Use-Cases wurde eine explorative Einzelfallstudie (Yin 2018) in einem deutschen Großunternehmen mit etwa 9000 Mitarbeitern durchgeführt. Fallstudien eignen sich, der Zielstellung dieses Beitrags entsprechend, besonders dazu, um innerhalb von Gebieten mit bisher wenig durchgeführten Studien zu forschen, vom aktuellen Stateof-the-Art zu lernen und ausgehend von der Praxis Hypothesen und Theorien zu generieren (Benbasat et al. 1987). Bei der Use-Case-Erstellung wurde sich an den Richtlinien des gestaltungsorientierten Forschungsparadigmas der Wirtschaftsinformatik (Hevner et al. 2004) orientiert.

Das Unternehmen wurde im Rahmen eines zwölfmonatigen Projekts zur Auswahl eines geeigneten Self-Service-Portals mit Schwerpunkt IT-Service-RequestManagement als Erweiterung seiner bestehenden ITSM-Software-Suite begleitet. Bislang wurde im Unternehmen eine begrenzte Anzahl an Use-Cases angeboten, die allerdings nicht vollständig standardisiert und transparent war. Angestoßen wurden die Use-Cases hauptsächlich per E-Mail, teilweise per Telefon. Im Zuge der Self-Service-Portal-Auswahl sollten relevante Use-Cases identifiziert und spezifiziert werden.

Zur Datenerhebung wurden im Zeitraum Januar bis Mai 2021 mehrere Halbtages-Workshops und zahlreiche Einzel-Experteninterviews durchgeführt. Teilnehmer waren erfahrene interne ITSM-, Vertriebs- und IT-Service-Katalog-Spezialisten, repräsentative spätere Portal-Nutzer sowie Vertreter von Herstellern und Dienstleistern führender ITSM-Software-Suiten und Self-Service-Portalen. Dabei wurden im Brainstorming-Verfahren funktionale und nicht-funktionale Anforderungen an ein Self-Service-Portal, insbesondere den Use-Cases betreffend, zusammengetragen. Jeder ermittelte Use-Case wurde bis auf Einzelschrittebene heruntergebrochen. Während den Datenerhebungssitzungen wurden ausführliche, handschriftliche Notizen angefertigt, die im Nachgang ausgearbeitet wurden. Die Use-Case-Beschreibungen wurden innerhalb des gesamten Teilnehmerkreises verteilt und in Nachfolgeterminen hinsichtlich ihrer grundsätzlichen Sinnhaftigkeit und, wenn gegeben, ihrer Form der Umsetzung umfassend diskutiert und iterativ überarbeitet. Beendet wurde das iterative Vorgehen, nachdem weder schriftlich noch mündlich Anpassungs- oder Erweiterungsvorschläge vorgebracht wurden. 


\section{Ergebnis: Use-Case-Katalog}

Aus Platzgründen kann in diesem Beitrag nicht der detaillierte Prozessablauf der einzelnen Use-Cases beschrieben werden. Stattdessen werden sie in diesem Kapitel aufgelistet und allgemein erläutert. Die Reihenfolge spiegelt dabei keine Prioritäten wider. Danach folgt ein zusammenfassendes Use-Case-Diagramm.

(1) Neubestellung: Mitarbeitern muss die Möglichkeit gegeben werden, die gewünschten IT-Services in der bevorzugten SLA-Variante auszuwählen, im Warenkorb zu sammeln und zu bestellen. Da für gewöhnlich nicht jeder Mitarbeiter berechtigt ist, sämtliche im Service-Katalog enthaltenen IT-Services zu beziehen, muss durch ein Rollenmodell sichergestellt sein, dass im Self-Service-Portal jeweils nur die entsprechende Teilmenge angezeigt wird.

(2) Kurzzeitiges Mieten: Als spezielle Form der Neubestellung sollte ein SelfService-Portal gestatten, bestimmte IT-Services für temporär stattfindende Veranstaltungen mit einem Preisaufschlag zu beziehen. Dazu zählen, je nach Unternehmen, beispielsweise mobile Endgeräte, WLAN-Access-Points und Beamer. Es empfiehlt sich, für kurzzeitig mietbare IT-Services eine eigene Kategorie innerhalb des ITService-Katalogs anzulegen.

(3) Onboarding: Als weitere Sonderform der Neubestellung sollte ein Self-Service-Portal Mitarbeitern mit Leitungsfunktion und deren Assistenten die Option bieten, im Rahmen des Onboarding-Prozesses für neue Mitarbeiter die notwendigen IT-Services in Form größerer, vordefinierter Basis-IT-Service-Pakete zu bestellen. Als Voraussetzung dafür muss ein Onboarding-Service-Portfolio, das etwa nach grundlegenden Mitarbeiterrollen, wie unter anderem Controlling- und IT-Mitarbeiter, gegliedert ist, definiert werden. Hierdurch lässt sich der Aufwand für ähnliche, wiederkehrende Bestellungen und die Gefahr, einzelne IT-Services zu übersehen, reduzieren.

(4) Kostenloser Leistungsabruf: Ein Self-Service-Portal sollte es ermöglichen, gewisse IT-Services zu einem Preis von null Euro anzubieten. Kostenlose Leistungsabrufe stehen vorwiegend für optionale Komponenten innerhalb aktuell bezogener IT-Leistungspakte. Darunter fallen beispielsweise Kleinteile wie Headsets und Kabel.

(5) Umzug: Neben der Neubestellung und deren Spezialformen sollte der Umzug von gegenwärtig in Anspruch genommenen IT-Services abgebildet werden. Erfahrungsgemäß kommt es regelmäßig vor, dass Mitarbeiter ihren Standort wechseln. Mitarbeiter sollten angesichts dessen über das Self-Service-Portal den zukünftigen Standort angeben können. Dieser Use-Case sollte gleichermaßen einen Umzug aller aktuell bezogener IT-Services (Umzug-Mitarbeiter) und einzelner IT-Services (Umzug-Einzelequipment) beinhalten. 
(6) Nutzerwechsel: Ein Self-Service-Portal sollte die Übertragung von bezogenen IT-Services auf einen anderen Abnehmer abdecken. Dieser Fall tritt zum Beispiel bei Abgang und gleichzeitigem Zugang eines Mitarbeiters ein.

(7) Gerätetausch (Hardware-Erneuerung): In das Self-Service-Portal sollte einbezogen werden können, dass der Nutzer nach einem bestimmten Zeitraum seine bisherige Hardware kostenlos gegen eine neuere Ausführung tauschen kann. Im ITService-Katalog bietet sich zu Informationszwecken das Attribut „Mindestnutzungsdauer" an.

(8) Variantenwechsel: Der Kunde sollte während der Vertragslaufzeit seine bisherigen IT-Services jeweils gegen eine alternative Ausführung (Beispiel: größeres oder kleineres E-Mail-Postfach) eintauschen können.

(9) Organisations- und Abteilungswechsel: Kunden sollten die Auswahlmöglichkeiten Organisations- (Wechsel von Mutter- zu Tochterunternehmen beziehungsweise zwischen Tochterunternehmen) und Abteilungswechsel vorfinden. Der Einfachheit halber genügt es gegebenenfalls in beiden Fällen, dem Kunden lediglich als Information anzuzeigen, dass er jeweils sämtliche aktuell bezogenen IT-Services kündigen und die für seine neuen Aufgaben benötigten IT-Services neubestellen muss.

(10) Mitarbeiterbeurlaubung und -abstellung: Ähnlich wie beim Use-Case Organisations- und Abteilungswechsel sollten dem Nutzer im Falle von Beurlaubung (unbezahlter Sonderurlaub) und zeitlich befristeter Abstellung eines Mitarbeiters (zu einer anderen Organisation innerhalb des Konzerns) zumindest dessen Handlungsmöglichkeiten im Self-Service-Portal eingeblendet werden.

(11) Stammdatenänderung: Der Kunde benötigt die Möglichkeit, über das SelfService-Portal seine Stammdaten zu prüfen. Dabei muss er bestimmte Stammdaten selbst pflegen können. Darunter fallen beispielsweise die Änderung des Namens und der Kostenstelle.

(12) Kündigung: Ein Kunde muss derzeit bezogene IT-Services auf Basis von klar definierten Regelungen kündigen können. Das Self-Service-Portal muss dem Kunden anzeigen, welche IT-Services er in Anbetracht seiner jeweiligen Vertragslaufzeiten und Kündigungsfristen aktuell kündigen könnte. Wenn ein IT-Service derzeit nicht kündbar ist, soll ausgewiesen werden, ab welchem Zeitpunkt dies frühestens möglich ist.

(13) Offboarding: Mitarbeitern mit Leitungsfunktion und deren Assistenten muss im Rahmen des Offboarding-Prozesses die Möglichkeit gegeben werden, die gegenwärtig bezogenen IT-Services des ausscheidenden Mitarbeiters unmittelbar, ohne Einhaltung von Kündigungsfristen, innerhalb des Self-Service-Portals kündigen zu können. Voraussetzung ist eine Gesamtübersicht über die von jedem direkt zugeordneten Mitarbeiter bezogenen IT-Services. Wünschenswert wäre eine Schnittstelle 
in Richtung Personalabteilung zur Informierung über den erfolgten OffboardingSchritt.

(14) Berechtigungsbestellung, -änderung und -kündigung: Neben der Use-Cases, welche die IT-Services im engeren Sinn betreffen, muss Selbiges für Berechtigungen (Active-Directory) möglich sein. Ein Beispiel ist der Wechsel eines Nutzers von einer einfachen zu einer privilegierten Kennung bei einer bestimmten Anwendung.

(15) Verlust- und Diebstahlmeldung: Mitarbeitern sollte die Möglichkeit gegeben werden, einen Verlust oder den Diebstall einer IT-Service-Komponente über das Self-Service-Portal zu melden und damit deren Sperrung zu veranlassen.

(16) Durchführung aller Use-Cases für andere Person: Im Self-Service-Portal sollten sämtliche Use-Cases im Namen einer anderen Person durchführbar sein. Dies betrifft in der Praxis vor allem Führungskräfte und deren Assistenten (Abteilungskostenstelle) sowie Projektleiter im Rahmen von Projekten (Projektkostenstelle).

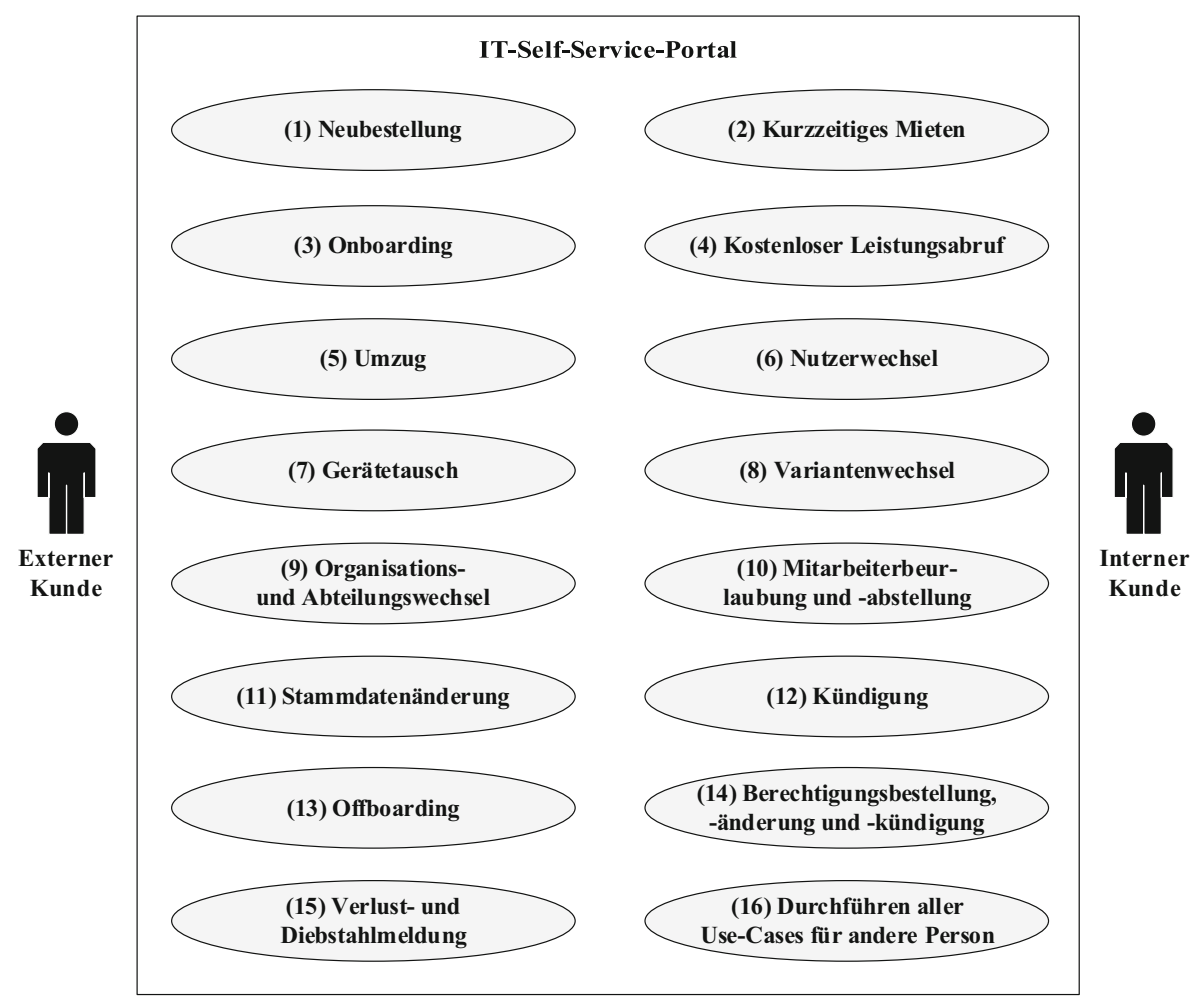

Abb. 1 Zusammenfassung der identifizierten Use-Cases 
Abb. 1 fasst die 16 Use-Cases in Form eines Use-Case-Diagramms zusammen. Zur Wahrung der Übersichtlichkeit wird auf die Assoziationen verzichtet. Akteure können sowohl interne als auch externe Kunden sein.

\section{Diskussion}

Im Rahmen dieser explorativen Einzelfallstudie konnten insgesamt 16 als relevant eingestufte Use-Cases für ein ITSM-Self-Service-Portal ermittelt werden. Diese zeigen, dass Mitarbeiter vielfältige Anliegen haben, die weit über die Neubestellung und Kündigung von IT-Services hinausgehen. Eingeschränkt werden muss hierbei, dass nicht jeder der Use-Cases in seiner Häufigkeit und wirtschaftlichen Bedeutung gleich relevant ist. Fälle wie Mitarbeiterbeurlaubung und -abstellung oder Organisations- und Abteilungswechsel kommen vergleichsweise seltener vor. Unternehmen müssen daher im Einzelnen prüfen, welche Use-Cases sie erstens grundsätzlich anbieten möchten und zweitens welche davon Bestandteil ihres Self-Service-Portals werden sollen. Eine unreflektierte Einbindung sämtlicher Use-Cases in ein Self-Service-Portal kann sogar ökonomisch nachteilig sein, da kein existierendes im Standard Tool alle abdeckt und deren individuelle Programmierung mit entsprechenden Kosten verbunden ist. Ein sinnvoller Kompromiss kann deshalb sein, bei komplexen beziehungsweise voraussichtlich selten in Anspruch genommenen Use-Cases einen Verweis auf einen alternativen Vertriebsweg, wie eine Telefonnummer oder E-MailAdresse, im Self-Service-Portal zu hinterlegen. Letztendlich kommt es darauf an, dass die Nutzer mitgenommen und geführt werden. Ansonsten besteht die Gefahr der Ablehnung des Self-Service-Portals. Ungeachtet dessen sollte bei Problemen und Unklarheiten weiterhin die Möglichkeit bestehen, das Auftragsmanagement telefonisch zu kontaktieren. Da sich Personen in ihrem Interaktionsbedürfnis unterscheiden, sollte ein Self-Service-Portal nicht als vollständiges Substitut zum persönlichen Vertriebsweg gesehen werden.

Self-Service-Portal-Einführungsprojekte gehen häufig mit der Erstellung beziehungsweise der Überarbeitung eines IT-Service-Katalogs einher. Liegt der IT-Service-Katalog in einer unzureichenden Qualität vor, ist ein Scheitern des Self-ServicePortals unausweichlich. Denn dann besteht das Risiko, dass die Mehrzahl der Kunden den bisherigen telefonischen oder schriftlichen Weg vorziehen. Unternehmen, der dieser Schritt Probleme bereitet, sollten Hilfe von einem darauf spezialisierten externen Dienstleister in Anspruch nehmen. Nach Vorliegen eines geeigneten ITService-Katalogs und der Auswahl und Spezifikation der Use-Cases muss geprüft werden, inwieweit die auf dem Markt verfügbaren Self-Service-Portale beziehungsweise das Modul der vorhandenen ITSM-Software-Suite diese Teilmenge umsetzen. Hierbei wird sich ein Customizing oder gar eine grundlegende Neuerstellung des Ablaufs in einem Self-Service-Portal kaum vermeiden lassen. Bei der Realisierung müssen die Fragen beantwortet werden, welche Daten für jeden Use-Case benötigt werden, inwiefern diese in ausreichender Qualität vorliegen und wer für deren Pflege verantwortlich ist. Zusammenfassend werden bei der Self-Service-Portal-Auswahl und -Einführung folgende vier Kernphasen durchlaufen (Abb. 2): 


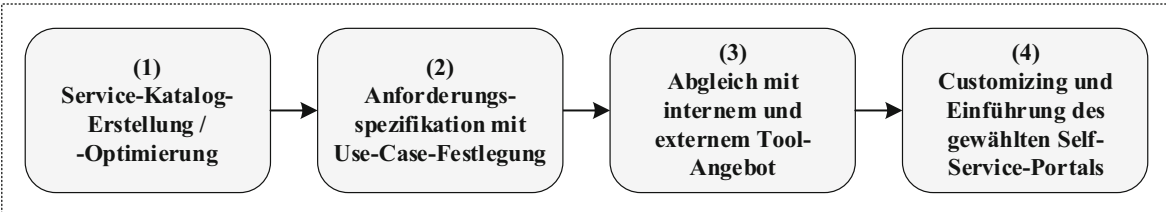

Abb. 2 Kernphasen der Self-Service-Portal-Auswahl und -Einführung

Selbstverständlich reicht die Gewährung der Auslösung eines Service-Requests über ein Self-Service-Portal nicht aus. Diese müssen seitens des IT-Bereichs entsprechend umgesetzt werden. Ein Service-Request sollte einen Workflow im ITSMKernsystem triggern. Ein Workflow legt fest, welche Aufgaben einzelne IT-Systeme und Personen in welcher Reihenfolge abarbeiten müssen, damit der jeweilige Use-Case umgesetzt und abgerechnet wird. Grundvoraussetzung ist ein hoher Grad an Integration von organisatorischen, kaufmännischen und technischen SoftwareTools. Während des Bereitstellungsprozesses erwarten Kunden eine aussagekräftige Statusanzeige im Self-Service-Portal. Für sämtliche Use-Cases müssen spezifische Genehmigungsworkflows definiert und hinterlegt werden. Ein Service-Request sollte erst dann bearbeitet werden, wenn die erforderliche Genehmigung seitens der dafür zuständigen Führungskraft oder deren Vertreters vorliegt.

Nachdem es wenig Sinn macht, für jeden ITSM-Prozess ein eigenes Kundenportal zu etablieren, stellt sich die Frage, welche neben dem Service-Request-Management abgebildet werden sollten. Hierbei ist zunächst das Rollen- und Rechtemanagement - Anlegung eines Nutzers und Zuweisung spezifischer Berechtigungen innerhalb eines bestimmten Systems - zu nennen (Use-Case 14). Da es unausweichlich irgendwann zu Störungen an IT-Services kommen wird, sollte das Incident-Management integriert werden. Viele der auf dem Markt erhältlichen Self-Service-Portale erfüllen diese Punkte. Weitere vielversprechende Kandidaten sind das Configuration(Übersichten von CIs) und das Demand-Management (Erstellen und Verfolgen von Demands). Wird der Fokus über Standardaufträge hinaus gerichtet, können Dinge wie Consulting- und Trainingsleistungen aufgenommen werden. Das Endziel sollte jedenfalls sein, ein zentrales Self-Service-Portal für möglichst viele IT-Belange zu erschaffen.

\section{Nutzen für Forschung und Praxis, Limitationen und Ausblick}

Die Ergebnisse dieser explorativen Einzelfallstudie sind sowohl für die Praxis als auch für die Forschung von Nutzen. Unternehmensentscheidern und IT-Verantwortlichen soll der erarbeitete Use-Case-Katalog als Entscheidungshilfe für die Self-Service-Portal-Auswahl und -Einführung dienen. Unternehmen, die bereits ein Self-Service-Portal einsetzen beziehungsweise planen, das innerhalb ihrer vorhandenen ITSM-Software-Suite integrierte zu verwenden, erhalten eine Referenz für mögliche Anpassungen und Erweiterungen. Denn keines der aktuell verfügbaren Self-Service-Portale deckt alle in dieser Studie ermittelten Use-Cases ab. Anbieter von Self-Service-Portalen beziehungsweise deren Partnerunternehmen bieten 
jedoch häufig die Möglichkeit kundenindividueller Anpassungen an. Etablierten Tool-Herstellern und Start-ups kann der Use-Case-Katalog als Basis für etwaige Erweiterungen und Verbesserungen dienen. Wem es gelingt, ein Self-ServicePortal zu entwickeln, das einen breiten Funktionsumfang bietet, dabei über eine hohe User-Experience verfügt, sich einfach in die Unternehmensarchitektur einbinden lässt und nicht an eine bestimmte ITSM-Software-Suite gebunden ist, hat gute Erfolgsaussichten.

Wissenschaftlern liefern die Ergebnisse zahlreiche Anknüpfungspunkte für weitere Forschung. Als nächstes könnten sie untersuchen, inwieweit die Use-Cases von den gegenwärtig auf dem Markt erhältlichen Self-Service-Portalen jeweils abgedeckt werden. Als Informationsquelle bieten sich die jeweiligen Internetauftritte an. Der Abgleich von Anforderungen und Angebot wäre für potenzielle Kunden für ihren Auswahlprozess von Nutzen. Gegebenenfalls lassen sich dabei weitere UseCases identifizieren. Denn die vorliegende Einzelfallstudie kann nicht den Anspruch erheben, sämtliche relevante Use-Cases ermittelt zu haben. In dem Zusammenhang könnte der Frage nachgegangen werden, inwieweit unterschiedliche Branchen und Unternehmensgrößen möglicherweise etwas anders gelagerte Use-Cases benötigen. Nicht zuletzt muss erforscht werden, wie die Use-Cases am sinnvollsten in einem Self-Service-Portal umgesetzt werden sollten.

Am Ende bleibt festzuhalten, dass die Forschung im Hinblick auf ITSM-SelfService-Portale noch relativ am Anfang steht. Dieser Beitrag trägt zwar mit seinen Ergebnissen dazu bei, die vorherrschende Situation zu verbessern, dennoch existieren noch einige weiße Flecken auf der Forschungslandkarte. Angesichts deren gegenwärtigen und im Rahmen der zunehmenden Digitalisierung voraussichtlich steigenden Bedeutung in der Praxis ist es an der Zeit, dass Self-Service-Portale stärker in den Fokus der Scientific-Community rücken.

Funding Open Access funding enabled and organized by Projekt DEAL.

Open Access Dieser Artikel wird unter der Creative Commons Namensnennung 4.0 International Lizenz veröffentlicht, welche die Nutzung, Vervielfältigung, Bearbeitung, Verbreitung und Wiedergabe in jeglichem Medium und Format erlaubt, sofern Sie den/die ursprünglichen Autor(en) und die Quelle ordnungsgemäß nennen, einen Link zur Creative Commons Lizenz beifügen und angeben, ob Änderungen vorgenommen wurden.

Die in diesem Artikel enthaltenen Bilder und sonstiges Drittmaterial unterliegen ebenfalls der genannten Creative Commons Lizenz, sofern sich aus der Abbildungslegende nichts anderes ergibt. Sofern das betreffende Material nicht unter der genannten Creative Commons Lizenz steht und die betreffende Handlung nicht nach gesetzlichen Vorschriften erlaubt ist, ist für die oben aufgeführten Weiterverwendungen des Materials die Einwilligung des jeweiligen Rechteinhabers einzuholen.

Weitere Details zur Lizenz entnehmen Sie bitte der Lizenzinformation auf http://creativecommons.org/ licenses/by/4.0/deed.de.

\section{Literatur}

Abdelkebir S, Maleh Y, Belaissaoui M (2017) An agile framework for ITS management in organizations. A case study based on devops. In: 2. International conference on computing and wireless communication systems Larache 
Axelos (2019) ITIL foundation: ITIL 4 edition, 4. Aufl. TSO, Norwich (English Edition)

Bause M, Landis M (2019) Wie servicezentriert ist Ihre IT wirklich: Ergebnisse einer Online-Umfrage in der DACH-Region. USU, Möglingen

Beims M, Ziegenbein M (2014) IT-Service-Management in der Praxis mit ITIL, 4. Aufl. Carl Hanser, München

Benbasat I, Goldstein DK, Mead M (1987) The case research strategy in studies of information systems. Manag Inf Syst Q 11(3):369-386

Betz C, Mckeon-White W (2019) The Forrester wave: enterprise service management, Q4 2019. Forrester, Cambridge

Brooks JM, Curti D (2014) Critical capabilities for IT service catalog tools. Gartner, Stamford

Doheny R, Andes K, Cleary M (2020) Magic quadrant for IT service management tools. Gartner, Stamford

Floerecke S (2020) Best-Practices für die Gestaltung von IT-Service-Katalogen und den Einsatz von SelfService-Portalen. HMD Prax Wirtschaftsinform. https://doi.org/10.1365/s40702-020-00702-y

Floerecke S (2021a) Self-Service-Portale mit Schwerpunkt Service-Request-Management: Analyse der Eignung existierender Software-Tool-Kategorien. HMD Prax Wirtschaftsinform. https://doi.org/10. 1365/s40702-021-00726-y

Floerecke S (2021b) Service-Request-Typen und ITSM-Prozesse richtig abdecken. Digit Bus Cloud 1/2021:34-35

Hevner AR, March ST, Park J, Ram S (2004) Design science in information systems research. Manag Inf Syst Q 28(1):75-105

Jäntti M (2013) Exploring self-service support methods in IT service management. In: 10. International conference on service systems and service management Hong Kong

Lohse M (2016) Von der Selbstbedienung zum Self-Service - Wege zur Integration von Prozessbeteiligten. HMD Prax Wirtschaftsinform 53(6):866-878

Macias CM, Alonso IA (2018) Review of proposals for the construction and management of the catalog of information technology services. IEEE Access 6:45335-45346

Macias CM, Alonso IA (2019) Proposal for the identification of information technology services in public organizations. Symmetry 11(10):1-32

Mann S (2017) Self-Service: Die positive Kundenerfahrung im Fokus. It Serv Manag 41:24-27

Nord F, Dörbecker R, Böhmann T (2016) Structure, content and use of IT service catalogs - empirical analysis and development of a maturity model. In: 49. Hawaii international conference on system sciences (HICSS) Manoa

Pröhl T, Zarnekow R (2019) Agilität bei der Einführung von IT-Servicemanagement: Lösung klassischer Herausforderungen mit agilen Methoden. HMD Prax Wirtschaftsinform 56(2):301-317

Scherer A, Wünderlich NV, Von Wangenheim F (2015) The value of self-service: long-term effects of technology-based self-service usage on customer retention. Manag Inf Syst Q 39(1):177-200

Schopf V (2017) Vom IT-Shop zum Businesstreiber - Servicekataloge im ITSM. It Serv Manag 41:38-43

Schorr F, Hvam L (2018) The use of design-science to define information content requirements for IT service catalogs. In: IEEE international conference on industrial engineering and engineering management Bangkok

Schulz C (2015) IT-Katalogmanagement: Steuerungsinstrument für den internen Hard- und Softwarebestand. OBJEKTspektrum 3:68-72

Tiemeyer E (2020) Handbuch IT-Management: Konzepte, Methoden, Lösungen und Arbeitshilfen für die Praxis, 7. Aufl. Carl Hanser, München

Yin RK (2018) Case study research: design and methods, 6. Aufl. SAGE, Thousand Oaks 